\title{
Switching Neuronal Inputs by Differential Modulations of Gamma-Band Phase-Coherence
}

\author{
Iris Grothe, ${ }^{1,2 *}$ Simon D. Neitzel, ${ }^{1,2 *}$ Sunita Mandon, ${ }^{1,2}$ and Andreas K. Kreiter ${ }^{1,2}$ \\ ${ }^{1}$ Brain Research Institute, Center for Cognitive Sciences, University of Bremen and ${ }^{2}$ Bernstein Group for Computational Neurosciences Bremen, University \\ of Bremen, D-28334 Bremen, Germany
}

Receptive fields (RFs) of cortical sensory neurons increase in size along consecutive processing stages. When multiple stimuli are present in a large visual RF, a neuron typically responds to an attended stimulus as if only that stimulus were present. However, the mechanism by which a neuron selectively responds to a subset of its inputs while discarding all others is unknown. Here, we show that neurons can switch between subsets of their afferent inputs by highly specific modulations of interareal gamma-band phase-coherence (PC). We measured local field potentials, single- and multi-unit activity in two male macaque monkeys (Macaca mulatta) performing an attention task. Two small stimuli were placed on a screen; the stimuli were driving separate local V1 populations, while both were driving the same local V4 population. In each trial, we cued one of the two stimuli to be attended. We found that gamma-band PC of the local V4 population with multiple subpopulations of its V1 input was differentially modulated. It was high with the input subpopulation representing the attended stimulus, while simultaneously it was very low between the same V4 population and the other input-providing subpopulation representing the irrelevant stimulus. These differential modulations, which depend on stimulus relevance, were also found in the locking of spikes from V4 neurons to the gamma-band oscillations of the V1 input subpopulations. This rapid, highly specific interareal locking provides neurons with a powerful dynamic routing mechanism to select and process only the currently relevant signals.

\section{Introduction}

One of the fundamental properties of sensory cortical pathways is the increase of receptive field (RF) sizes along successive processing stages, resulting from the convergence of afferent input from one stage to the next (see Fig. 1A). In the visual system, for example, RFs increase from tiny spots with diameters often less than a degree of visual angle in V1, to regions comprising large parts of the visual field in inferotemporal or parietal cortex (Gattass et al., 2005). This raises the problem that neurons beyond V1 often receive signals representing features of multiple independent objects, which are present in their RFs simultaneously and, therefore, compete for being processed (Reynolds et al., 1999). Attending one of multiple objects resolves this competition and results in neurons responding as if only the attended object was present (Moran and Desimone, 1985; Treue and Maunsell, 1996; Chelazzi et al., 1998; Reynolds et al., 1999). This is a remarkable capability as it requires a neuron to respond selectively to signals

\footnotetext{
Received Feb. 23, 2012; revised Sept. 6, 2012; accepted Sept. 14, 2012.

Author contributions: S.M. and A.K.K. designed research; I.G. and S.D.N. performed research; I.G. and S.D.N. analyzed data; I.G. and A.K.K. wrote the paper.

This work was supported by the Bernstein Group for Computational Neuroscience Bremen Grant 01GQ0705. I.G. was supported by the Leibniz Graduate School for Primate Neurobiology. We are grateful to K. Thoß, R. Hakizimana, and K. Taylor for monkey care and training; to W. Zinke for help with the anatomical MRIs; to U. Ernst for providing us with the expected value derivation; and to $\mathrm{U}$. Ernst and $\mathrm{D}$. Wegener for comments on the manuscript.

*I. Grothe and S.D. Neitzel contributed equally to this work.

The authors declare no competing financial interests.

Correspondence should be addressed to Dr. Andreas K. Kreiter, Brain Research Institute, Department of Theoretical Neurobiology, University of Bremen, FB 2, P.0. Box 330440, D-28334 Bremen, Germany. E-mail: kreiter@brain.uni-bremen.de.

DOI:10.1523/JNEUROSCI.0890-12.2012

Copyright $\odot 2012$ the authors $\quad 0270-6474 / 12 / 3216172-09 \$ 15.00 / 0$
}

from a small subset of afferents carrying the signals representing the attended object while rendering a larger number of possibly highly active inputs ineffective. Furthermore, it requires great flexibility in selecting a particular subset, since the position and properties of the attended stimulus and hence the composition of the subset of afferent inputs carrying the relevant signals can vary arbitrarily and quickly.

A candidate mechanism that may fulfill these requirements is gamma-band oscillatory synchronization. It has been shown that attention modulates gamma-band synchronization of anatomically connected neurons located within the same cortical area (Fries et al., 2001; Taylor et al., 2005) as well as in different areas (Saalmann et al., 2007; Gregoriou et al., 2009) when the neurons respond to a single stimulus present within their overlapping RFs. In contrast, a much more specific pattern of dynamic interactions is required for a mechanism by which a neuron can switch between subsets of its synaptic inputs, providing signals from multiple different stimuli in its RF. Accordingly, theoretical studies predict that this requires the receiver neuron to synchronize selectively with the subset of the input population representing the attended stimulus (Masuda, 2009; Tiesinga and Sejnowski, 2010). Simultaneously, the receiver neuron should desynchronize with its other, highly active, oscillatory inputs representing nonattended stimuli.

To test for this, we trained two monkeys on a shape-tracking task (Fig. 1B). The monkeys had to attend to one of two sequences of morphing shapes and detect the reappearance of the shape that was cued at the beginning of the trial. The two small stimuli were driving two separate local V1 populations, while they were driving the same local V4 population. Attention could therefore be 

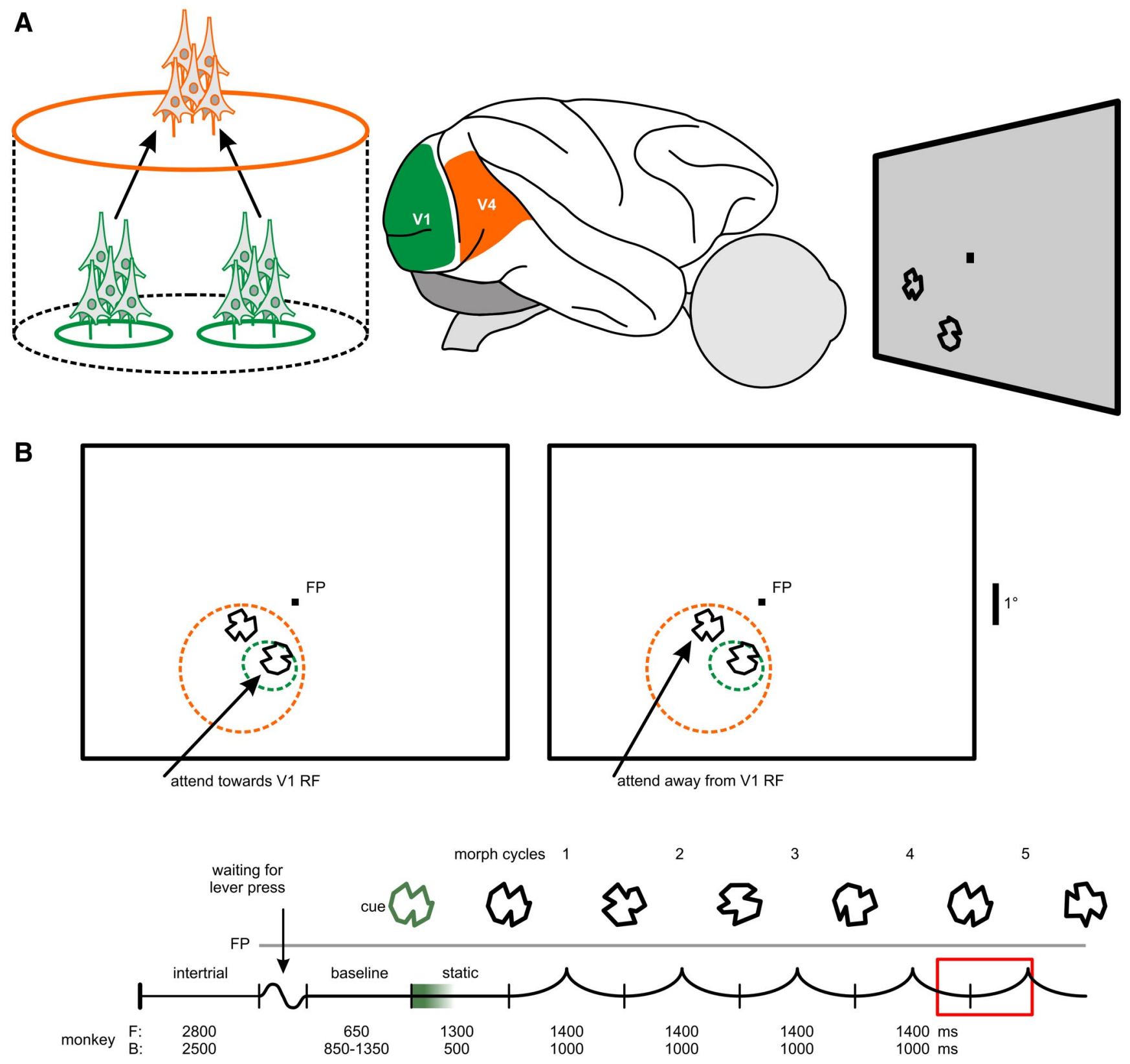

Figure 1. Simultaneous recordings in V1 and V4 during a shape-tracking task. $A$, Convergence of afferent inputs, which carry different signals competing for processing by the same neurons (left). Schematic of experimental constellation implementing competition between two nearby stimuli and cortical areas recorded while the monkeys performed the task (right; everywhere $V 1$ in green, V4 in orange). $\boldsymbol{B}$, Example of a recording constellation with RFs for V1 and V4 and arrows indicating attention (both not displayed on screen) directed toward the stimulus driving the recorded V1 site (left, upper row) or the competing distracter stimulus (right, upper row, corresponding data shown in Fig. $2 A$ ). Timeline for an example trial with target reappearance in MC 4 (bottom row). The red box indicates the response window, the gray line the presence of the fixation point (FP) on the screen. Note that only the target shape stream is shown here for simplicity.

directed toward or away from a particular V1 RF, whereas it was always directed toward the V4 RF. This stimulus configuration allowed us to examine whether the effective strength of the connections (Aertsen et al., 1989; Womelsdorf et al., 2007) between V4 neurons and the two subpopulations of their V1 input can indeed be differentially modulated.

\section{Materials and Methods}

Surgical preparation. Two adult male rhesus monkeys (Macaca mulatta) were implanted under aseptic conditions with a post to fix the head, a chronically implanted array over $\mathrm{V} 1$, and a recording chamber placed over area V4. The V1 array of monkey F, consisting of 96 tungsten electrodes with $2 \mathrm{~mm}$ spacing, was placed intracortically. Note that despite intracortical placement the electrodes record local field potentials (LFPs) only. Monkey B was implanted with a 128 electrode epidural array, fabrication as described by Rubehn et al. (2009). Its platinum electrodes (300 $\mu \mathrm{m}$ diameter) had an interelectrode spacing of $1.65 \mathrm{~mm}$. Localization of the cortical areas was based on anatomical magnetic resonance imaging scans obtained before surgery. All procedures and animal care were in accordance with the regulation for the welfare of experimental animals issued by the federal government of Germany and were approved by the local authorities.

Behavioral task. Monkeys were trained on a demanding shape-tracking task. If any parameter was different between the monkeys, parameters of monkey $\mathrm{B}$ are mentioned in the text and parameters of monkey $\mathrm{F}$ follow between brackets.

Visual stimuli were presented with a refresh rate of $100 \mathrm{~Hz}$ on a $22 \mathrm{inch}$ CRT monitor containing $1152 \times 864$ pixels $(1024 \times 768$ pixels $)$, which 
was placed at a distance of $92 \mathrm{~cm}(87 \mathrm{~cm})$ in front of the monkey. Eye position was monitored using a custom-made video eye tracking system (IScan; IScan Inc.).

The monkeys initiated a trial (Fig. $1 B$ ) by pressing a lever after the appearance of a central fixation point and they had to keep their gaze within a circular fixation window (diameter $1-1.5^{\circ}$ ) throughout the trial. After a blank period of $800-1350 \mathrm{~ms}$ (650 ms), two differently shaped, complex stimuli appeared in the lower right (lower left) quadrant of the visual field and were presented statically for $500 \mathrm{~ms}$ (1300 ms). To direct attention to the behaviorally relevant stimulus stream, two different cueing strategies were used. For monkey B, a cue was presented during the intertrial interval; it was the target shape at the target position, surrounded by a ring. For monkey F, the cue was presented in the first 200 ms of static shape presentation as a weak green color of the stimulus that faded away within the next $400 \mathrm{~ms}$. After static presentation, both the target and distracter shape started morphing into other shapes as described by Taylor et al. (2005). The cued initial shape reappeared at pseudorandomly selected positions in the sequence of shapes. The amount of morph cycles (MCs) could vary from 2 to 4 (2 to 5 ) and one MC lasted for $1000 \mathrm{~ms}$ (1400 ms). Recurrence of the initial shape in the cued shape stream required the monkey to release the lever to be rewarded with a small amount of fruit juice. A reappearance of the initial shape in the distracter stream had to be ignored. If the monkeys broke fixation, or responded too early or too late, the trial was aborted without reward. The response window ranged from $400 \mathrm{~ms}$ before the end of an MC to $350 \mathrm{~ms}(750 \mathrm{~ms})$ after the beginning of the next cycle.

Stimulus design and placement was led by the goal to create an experimental situation in which two local groups of V1 neurons provide competing input to $\mathrm{V} 4$ neurons, and to achieve a particularly challenging competition. Therefore, the two stimuli were very similar and closely spaced; each driving a local V1 population while driving the same V4 population. The $\mathrm{V} 1$ neurons at both recording sites are expected to provide input to the same V4 neurons, albeit indirectly via V2 (Gattass et al., 2005; Ungerleider et al., 2008; Markov et al., 2011). Within a recording session, stimuli were placed at equal eccentricity, in a range between 1.3 and $3.8^{\circ}$. Care was taken to achieve approximately equal responses from the V4 recording site for both stimuli to make sure they were placed at similarly responsive positions of the RF. Note that although attention could be directed toward or away from a V1 RF, it was always directed toward the V4 RF.

Recording. In V1, LFPs were recorded from the chronically implanted arrays to cover multiple putative recording sites at the same time. Note that despite the large number of electrodes used for this purpose, the size of V4 RFs and the spacing of V1 electrodes $(1.65-2 \mathrm{~mm})$ predict that only a few electrodes are expected to record from populations having their RFs at the two nonoverlapping and isoeccentric visual field locations that fit into a given V4 RF. Simultaneously, spikes and LFPs were measured from V4 with 1-3 epoxy-insulated tungsten micro-electrodes (125 $\mu \mathrm{m}$ diameter; $1-3 \mathrm{M} \Omega$ at $1 \mathrm{kHz}$; Frederic Haer) in the upper cortical layers. Reference and ground electrodes for monkey $\mathrm{F}$ were platinum-iridium wires below the skull at frontal and lateral sites. The reference for monkey B was placed on top of the epidural array, and the ground was a titanium pin at the posterior end of the skull. RFs were mapped manually while the monkey was fixating centrally, often followed by an automated mapping procedure consisting of rapid presentation of circular dots. Each electrode's signal was amplified $1000 \times(5000 \times$; PGA 64, Multi Channel Systems $\mathrm{GmbH}$ ) and digitized at $25 \mathrm{kHz}$.

Data analysis. To suppress common source effects, we either rereferenced the V1 or the V4 electrodes. For monkey $\mathrm{F}$, the V1 recording sites were re-referenced by subtracting the averaged raw signals of 35 functional V1 recording sites, which were not visually stimulated. Based on the distributions of power in the relevant gamma and lower frequency bands (see Results) during MC 2 and 3 for these 35 electrodes, we confirmed for each session that the average reference was not dominated by any one of the channels. For monkey B, the V4 recording sites were re-referenced to a local reference recorded by a conventional, low impedance electrode placed on top of the dura near the V4 recording site on a daily basis. Subsequently, signals were filtered between $0.3-12.2 \mathrm{kHz}$ (FIR filter) to obtain spike data. Spikes were detected by setting an am- plitude threshold interactively after off-line visualization (typically 2-3 SD above the mean), and we used spike sorting based on principal components (Abeles and Goldstein, 1977) and waveform characteristics such as peak time and amplitude to isolate multi-unit activity (MUA) and single-unit activity (SUA). Signals were filtered between 1-150 Hz (FIR filter) and downsampled to $1 \mathrm{kHz}$ for the LFP data. Forward and backward filtering was applied to avoid phase shifts. For the epidural LFP data, we computed the current source density (units of $\mathrm{V} / \mathrm{m}^{2}$ ) to suppress the effect of the common reference and to minimize spatial smearing (Nunez et al., 1997) as described by Taylor et al. (2005). For analyses in the time-frequency (TF) domain, the data were than convoluted with complex Gaussian Morlet's wavelets (Taylor et al., 2005).

All subsequent analyses were performed on MC 2 and 3 . The first MC is not relevant for the monkey, because the second shape can never be the target. The last MC is also not relevant: if the target figure did not appear until then, it will definitely follow subsequently. Response strength to the visual stimuli was calculated for all sites as part of recording pairs in a session. The power spectral density (PSD) was computed by taking the square of the absolute value of the result of the wavelet convolution and dividing it by the Nyquist frequency $(500 \mathrm{~Hz})$. Visual responses of LFPs and spikes were calculated as the average gamma-band response and spike rate, respectively, during MC 2 and 3 with respect to a baseline taken in the $800 \mathrm{~ms}(650 \mathrm{~ms})$ before stimulus onset by first subtracting baseline activity from the stimulus-driven activity and then dividing by baseline.

For all statistical comparisons between the attentional conditions, the Wilcoxon signed rank test was used. We tested always at a critical $\alpha$ level of 0.05 .

LFP-LFP phase-coherence analysis. For each TF bin, phase-coherence (PC) was calculated (based on the phase-locking value; Lachaux et al., 1999) as follows:

The wavelet transformation resulted in complex coefficients, which can be written in terms of their amplitude A and phase $\varphi$ as follows:

$$
\widetilde{x}_{j}^{n}(t, f)=A_{j}^{n}(t, f) e^{i \varphi_{j}^{n}(t, f)},
$$

Where $\tilde{x}_{j}^{n}(t, f)$ denotes the time- and frequency-dependent wavelet coefficient from electrode $j$ measured during trial $n$.

PC TF spectra were obtained by averaging the phase difference estimates in the complex plane across $N$ trials and taking the absolute value.

$$
P C(t, f)=\left|\frac{1}{N} \sum_{n} e^{i \varphi_{j}^{n}(t, f)-i \varphi_{k}^{n}(t, f)}\right| .
$$

PC measures the consistency of the phase difference between V1 and V4 over trials and ranges between 0 and 1 .

For a comparison of the PC between the attentional conditions, we averaged the $\mathrm{PC}$ values in a specific TF window. The time range was composed of MC 2 and 3, whereas the frequency range was selected based on the full-width at half-maximum (FWHM) of the mean PC spectrum, obtained by averaging PC TF spectra over all included sessions and collapsing over time (monkey F: $47.09-78.39 \mathrm{~Hz}$, monkey B: $52.14-86.80 \mathrm{~Hz}$ )

Note that the PC is positively biased. To correct for this, we subtracted the expected value (EV) from the measured PC. The EV quantifies the mean PC observed if all phase differences in Equation 2 were drawn from a uniform distribution $[0,2 \pi]$, thus being equivalent to a random walk in the 2D complex plane. Using the result from Sun and Yang (1992, their Eqs. 3 and 13), the EV in dependence on total number of trials $N$ is given by the following:

$$
E V(N)=\frac{\sqrt{ } \pi}{2 \sqrt{N}} .
$$

Spike-LFP coherence analysis. LFPs were recorded from V1 and V4, while spikes were obtained only in V4. Therefore, we created V1 LFP spike-triggered averages (STAs) by cutting segments of LFP data around the spike times $( \pm 100 \mathrm{~ms})$ of all V4 spikes in MCs 2 and 3. To quantify the spike-field coherence (SFC), the STA was wavelet transformed as described above and normalized by the average of the wavelet- 
transformed segments around spike time, resulting in a TF representation of the SFC (equivalent to the common procedure to calculate SFC; Fries et al., 2001). The SFC estimates how well spikes lock to the LFP and ranges between 0 and 1 . For all analyses, we averaged the SFC values in a specific TF window of the TF representation of the SFC, centered on spike time. The time and frequency ranges were selected based on the FWHM of the SFC averaged over all included sessions, collapsed over time to find the frequency range of interest (monkey F: 42.52-78.39 Hz, monkey B: $52.14-96.11 \mathrm{~Hz}$ ), and collapsed over frequencies to find the time interval of interest (monkey F: -30 to $+10 \mathrm{~ms}$ around spike time, monkey B: -27 to $+5 \mathrm{~ms}$ around spike time).

Also the SFC is positively biased and the values were bias corrected as proposed by Grasse and Moxon (2010):

$$
S F C_{B C}=\frac{N_{\text {spikes }} S F C-1}{N_{\text {spikes }}-1},
$$

where $S F C_{B C}$ is the bias corrected SFC, and $N_{\text {spikes }}$ is the number of spikes from which the SFC is calculated.

Inclusion criteria. The logic of our approach is that we create an experimental condition in which two local groups of V1 neurons provide competing input to V4 neurons (Fig. 1A). For this experimental approach, three requirements need to be fulfilled: (1) the stimuli should drive the local populations well and hence induce a gamma-band response in both the V1 and V4 recording sites, (2) a V1 recording site should be driven by only one of the two stimuli (i.e., no cross-activation of V1 sites), and (3) the V4 site should be driven by both stimuli (i.e., a similar response indicates both stimuli are driving the V4 site in a comparable manner and ensures that competition is present). Thus, we confined the analyses to recording pairs fulfilling the following set of criteria. First, the stimulus-induced gamma-band response in both attentional conditions was required to be $\geq 1$ (monkey $\mathrm{F}$ ) or $\geq 0.3$ (monkey B) in $\mathrm{V} 1$ and $\geq 1$ in V4. Note that the different V1 thresholds between the monkeys are due to the different recording techniques. Second, to make sure that the V1 site of a V1-V4 pair was driven predominantly by the corresponding stimulus, the gamma-band response to this stimulus had to be $>2.5$ times higher than the response to the stimulus placed outside the RF. Third, we restricted our analyses to those pairs in which the V4 recording site was driven similarly by the two stimuli; V4 sites with sustained responses to the two individual shape stimuli differing by more than a factor of 2.5 were excluded.

Correspondingly, for the spike-field analysis we confined the analyses to pairs with a visual response in V1 as described above and with a response in the $\mathrm{V} 4$ firing rate $\geq 0.5$. Minimal absolute firing rate during the response had to be $10 \mathrm{~Hz}$. While these criteria selected those recording sites where the stimuli induced a visual response, we again made sure that the V1 site of the V1-V4 pair was not influenced by the other stimulus, and that both stimuli were equally well driving the V4 site as described above, but now for the V4 site with respect to the firing rate response.

After calculating the PC and SFC for all recording pairs that fulfilled the above-mentioned criteria, we tested for both attentional conditions separately if the observed interareal coherence was significant. In at least one of both attentional conditions significant PC was required. For the PC, significance was assessed by testing whether phase differences clustered at a particular angular value or tended to be distributed uniformly across trials. To do so, we calculated the mean phase-difference angle of the gamma-band range within MC 2 and 3 for each single trial. For $N$ trials, this resulted in $N$ phase difference angles. The Rayleigh test assesses if these angles are uniformly distributed between $[0,2 \pi]$, and is based on the mean vector length of the phase difference distribution (Zar, 1999). If this $p$ value was smaller than the critical $\alpha$ level 0.05 ( $p<0.05)$, we then considered the LFPs of the pair to be significantly coherent.

For the SFC, significance was assessed by creating a surrogate distribution in which possible relationships between V4 spikes and V1 gamma-band LFP were disrupted by randomly jittering each spike within $\pm 25 \mathrm{~ms}$ around its original spike time and calculating the SFC as described above (repeated $1000 \times$ ). The median of the resulting distribution resembles the bias as calculated in Equation 4. We calculated the proportion of surrogates that resulted in a larger SFC value than the experimentally determined SFC value. If this $p$ value was smaller than the critical $\alpha$ level $0.05(p<0.05)$, the estimated SFC was then considered significant.

Procedure for trial selection to obtain similar or opposite V1 gamma-band $P S D$ in both attentional conditions. For a control analysis we aimed to have similar V1 gamma-band PSD for both attentional conditions at each V1 recording site. To do so, we calculated the V1 gamma-band PSD for each trial, subtracted the average baseline response from the response during MCs 2 and 3, and then divided by this baseline. Subsequently, the trials were binned according to their gamma-band PSD for both attentional conditions separately (gamma-band frequency range: monkey F: 47.09-78.39 Hz, monkey B: $52.14-86.80 \mathrm{~Hz}$ ). We selected only trials from the overlapping part of the distributions and made sure to take an equal amount of trials for both attentional conditions from each bin.

In a related approach, we selected trials based on the single-trial V1 gamma-band PSD to test the extreme case in which the stimulus, when attended, induced only weak gamma-band PSD, whereas when attention was away, the stimulus induced high gamma-band PSD. For this purpose, we selected the trials with highest gamma-band PSD for the condition when the stimulus was not attended and the trials with lowest gamma-band PSD in the condition when the stimulus was attended, up until (but not including) the trial in which the responses became equal or opposite. Only stimulus-induced responses above the V1 PSD thresholds were considered.

\section{Results}

After training, the monkeys were well performing on the attentionally demanding shape-tracking task. Excluding trials terminating with an eye error, monkey $\mathrm{F}$ performed with $74.1 \%$ correct, 9.7\% early, and $16.2 \%$ late responses; monkey B performed with $92.6 \%$ correct, $5.2 \%$ early, and $2.2 \%$ late responses averaged over all sessions included in the analyses (monkey F: $33.7 \%$, monkey B: $26.7 \%$ eye errors). LFP data for $\mathrm{V} 1-\mathrm{V} 4$ pairs were acquired in 35 sessions ( 20 in monkey B, 15 in monkey F) providing between one and six pairs. From 87 pairs in which both sites responded sufficiently well to the stimuli, 45 pairs (out of 10 V1 sites and 36 V4 sites; each providing a median of 232 trials, range 124-483) fulfilled the full set of critical experimental conditions: clear stimulus responses of the V4 site to each of the two stimuli with similar strength and selective responses of V1 sites to only one of both stimuli (see Materials and Methods). Correspondingly, for the spike-field analysis, from 34 sufficiently responding V1-V4 pairs recorded in 16 of 21 sessions, 22 pairs fulfilled all conditions (out of $6 \mathrm{~V} 1$ sites and $16 \mathrm{~V} 4$ sites; each providing a median of 295 trials, range 124-469). An example of the typical arrangement of the V1 population RF (about $1-2^{\circ}$ in diameter) and the V4 population RF is shown in Figure $1 B$.

\section{Local V4 populations selectively synchronize with behaviorally relevant $\mathrm{V} 1$ inputs}

We analyzed the electrophysiological data recorded while the monkeys were performing the shape-tracking task and found that when two V1 subpopulations deliver signals to a single V4 population about different stimuli simultaneously, gamma-band PC between the local V4 population and its two input-providing V1 subpopulations is differentially modulated. The direction of the modulation depended strongly on which stimulus was behaviorally relevant. An example from monkey $\mathrm{F}$ is shown in Figure $2 \mathrm{~A}$, demonstrating that gamma-band $\mathrm{PC}$ between the respective $\mathrm{V} 1$ and $\mathrm{V} 4$ population was strong when attention was directed toward the stimulus driving the recorded V1 site (Fig. $2 \mathrm{~A}$, left; PC: 0.097). For the same V1 and V4 population PC was low (Fig. $2 A$, right; PC: 0.021 ) when attention was directed away from the V1 RF but still toward the V4 RF. A second example depicts a session with both V1 subpopulations recorded at the same time (Fig. 2 B, 
monkey B). It illustrates the simultaneous presence of entirely opposite strengths of gamma-band PC between V4 and its two different input subpopulations delivering relevant and irrelevant signals. When attention was directed toward the right stimulus (Fig. 2B, left column) PC between the V4 population and the V1 population providing the behaviorally relevant signals was more than a factor of six higher (Fig. 2 B, upper left; PC: 0.102) than between the same V4 population and the $\mathrm{V} 1$ population representing the irrelevant left stimulus (Fig. $2 \mathrm{~B}$, lower left; PC: $0.016)$. However, when attention was directed toward the left stimulus (Fig. $2 B$, right column), the pattern of effective connectivity reversed dynamically with PC now being more than a factor of seven stronger for the pathway providing signals from the now attended left stimulus (Fig. $2 B$, lower right; PC: 0.065$)$ compared with the other one (Fig. 2B, upper right; PC: 0.009).

Across V1-V4 recording pairs and monkeys $(n=45)$, the pattern of attention-dependent modulation of the interareal gamma-band PC was similar to the examples and the difference between the conditions was highly significant (Fig. $2 C, D$; Wilcoxon signed rank test, $p<$ $\left.10^{-4}\right)$. The median interareal PC was more than a factor of four higher when the V1 subpopulation carried the relevant signal (attend toward V1 RF, PC: 0.086, range $0.011-0.168$; attend out $\mathrm{V} 1 \mathrm{RF}, \mathrm{PC}$ : 0.018 , range $0.001-0.100$ ). Almost without exception, the recording pairs depicted in Figure $2 C$ show that the PC between a population in V4 and one of its input-providing V1 subpopulations was higher if attention was directed toward the stimulus driving this V1 subpopulation. Often, when attention was directed away from this driving stimulus the interaction came close to an almost complete shutdown compared to when attention was directed toward the driving stimulus. This strong attention-dependent difference in V1-V4 PC was specific to the gamma band.

The results (Fig. 3) show that attention does not modulate PC between V1 and V4 in the lower frequencies around the alpha/beta band (strongest attentional power modulation at $13.9 \mathrm{~Hz}, \mathrm{PC}$ analysis window: 8.9-18.9 Hz): when attention was directed toward the stimulus driving the $\mathrm{V} 1$ recording site the median PC was 0.029 (range: -0.013-0.094); whereas when attention was directed toward the other stimulus not driving the V1 site, median PC was 0.028 (range: $-0.002-0.090$; Wilcoxon signed rank test, $p=0.29$ ).

V1 power changes do not underlie selective V1-V4 PC modulations

It has been shown that attention increases gamma-band synchrony in visual cortex (Fries et al., 2001; Taylor et al., 2005; for
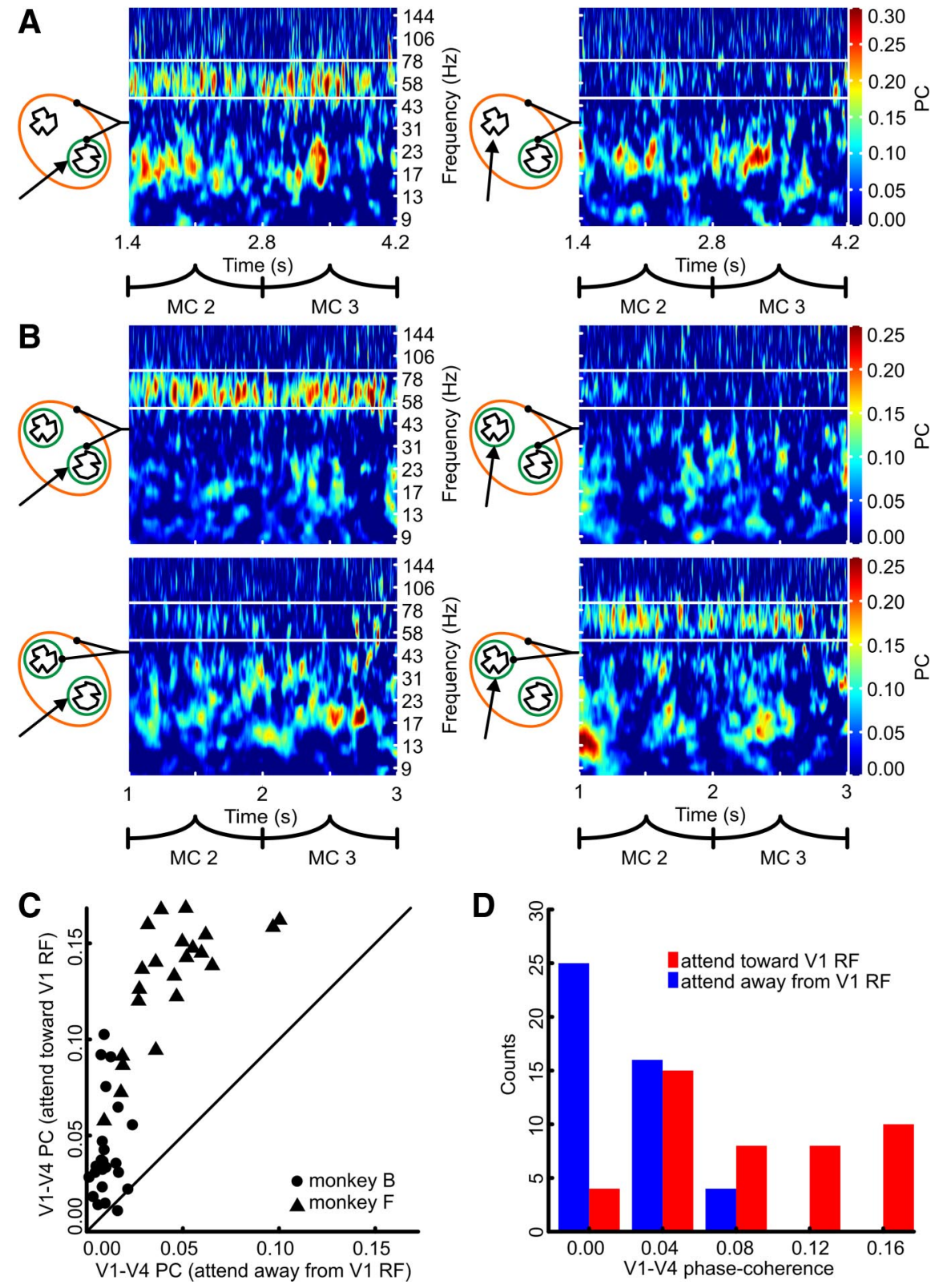

Figure 2. Attention differentially modulates gamma-band PC between multiple V1 subpopulations and V4. $\boldsymbol{A}$, Example of monkey F. Black lines originating from the TF plot connect to the symbolically depicted constellation on which the TF plot was ma-band $P C$ difference between the attentional conditions. Each dot and triangle represents a single recording pair. $\boldsymbol{D}$ Histograms of the gamma-band PC for the two attentional conditions (pooled data of both monkeys).

review, see Chalk et al., 2010 for an opposite effect in V1). Therefore, we analyzed whether the observed changes in interareal PC might be only due to attention-dependent modulations of gamma-band power in V1. In V4, due to the high similarity of the stimuli and their combined placement within an RF, the popluations were driven similarly well by both stimuli; sessions not conforming to this constellation were excluded from the analysis (see Materials and Methods). However, in V1, attention was directed either toward or away from the RF, thereby possibly changing the local gamma-band power in the V1 populations. A related concern is that small gaze drifts of the monkey could lead to such differences in $\mathrm{V} 1$ power by differentially stimulating either one or the other population more. We, therefore, repeated 


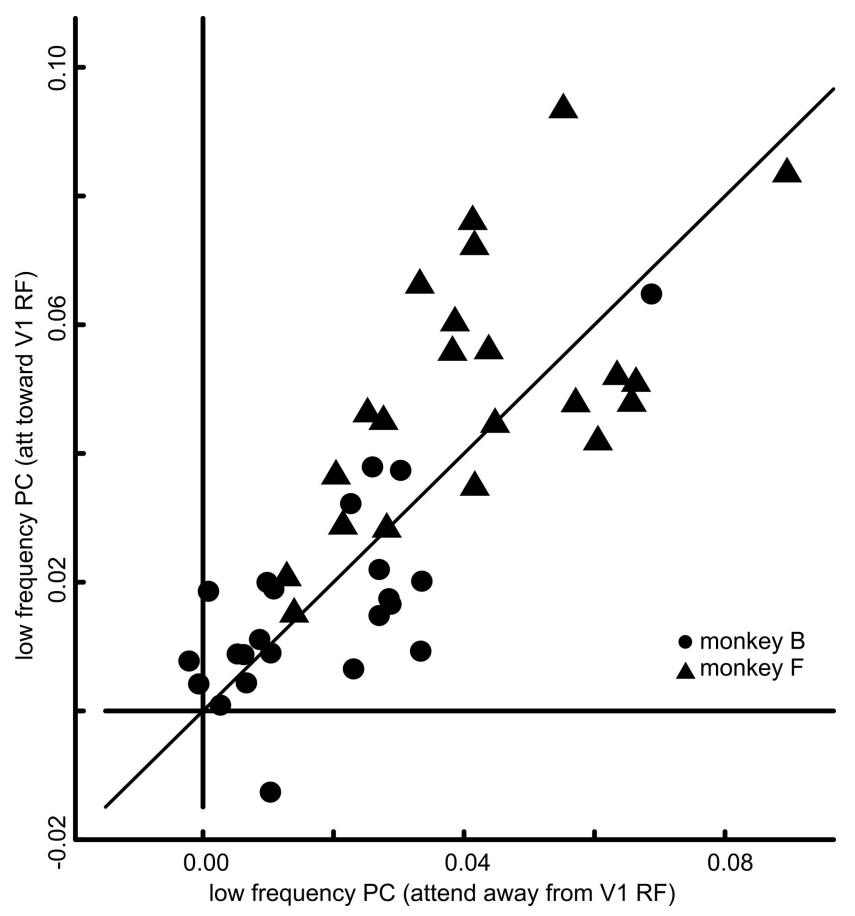

Figure 3. No attentional modulation of V1-V4PC in the alpha/beta band. Scatter plot showing gamma-band PC difference between the attentional conditions. Each dot and triangle represents a single recording pair $(n=45)$.

the analysis on a selected subset of trials with similar gammaband power in V1 (Fig. 4A). When attention was directed toward the stimulus driving the V1 site the median PSD was 0.980 (range $0.350-3.342$ ); when attention was directed toward the other stimulus it was 0.910 (range 0.383-3.373; Wilcoxon signed rank test, $p=0.19$ ). Also, when only this subset of trials with similar V1 PSDs was considered, V1-V4 PC was clearly stronger if attention was directed toward the stimulus driving the corresponding V1 site [Fig. $4 B$; attend stimulus driving V1 site, median PC: 0.055 (range 0.005-0.147); attend other stimulus, median PC: 0.015 (range 0.001-0.098); Wilcoxon signed rank test, $p<$ $\left.10^{-4}\right]$. In addition, we tested the highly unbalanced case with trials selected such that the attended stimulus always induced a PSD at the V1 site lower than the weakest selected response for the nonattended stimulus. Since the origin of such trials with particular low PSD is unclear (among the possible reasons is a temporary misallocation of attention), the result needs to be treated carefully. However, even in this extreme scenario, the V1-V4 PC was stronger if attention was directed toward the stimulus driving the corresponding V1 site [attend toward V1 RF, median PC: 0.033 (range $-0.004-0.100$ ); attend away from V1 RF, median PC: 0.018 (range $-0.007-0.088$ ); Wilcoxon signed rank test, $p<10^{-4}$.

V4 neurons selectively process behaviorally relevant inputs by differential coupling of their spikes to gamma-band LFP of the V1 input subpopulations

Selecting the behaviorally relevant inputs while ignoring the irrelevant ones is not only useful for computations performed by local populations of neurons but is an indispensable requirement for the signal processing done by individual neurons. Therefore, we subsequently tested whether spiking activity of individual V4 neurons also locks selectively to the gamma-band activity of the V1 population providing the behaviorally relevant information by calculating the SFC between V4 SUA and MUA activity and V1 LFPs.

Even though V1 and V4 are only scarcely linked by monosynaptic connections (Gattass et al., 2005; Ungerleider et al., 2008; Markov et al., 2011), we observed that spikes of neurons in V4 could be precisely phase locked to the gamma-band oscillations of the V1 LFP. Figure 5A shows two example V1 LFP STAs based on MUA (Fig. 5A, upper row, monkey F) and SUA (Fig. 5A, lower row, monkey B) in V4. In both cases, the different gamma-band amplitudes of the STAs show that the spike-field locking between V4 spikes and the V1 LFP were stronger with the V1 subpopulation carrying the behaviorally relevant information than with the V1 subpopulation carrying the irrelevant information. This difference was quantified using the TF representations of the SFC (Fig. 5B), which show an increase by more than a factor of four for the MUA-based STA (attend toward V1 RF, SFC: $7.749 \cdot 10^{-4}$, attend out V1 RF, SFC: $1.789 \cdot 10^{-4}$ ) and an increase in SFC by a factor of 16 for the SUA-based STA (attend toward V1 RF, SFC: $1.71 \cdot 10^{-3}$, attend out V1 RF, SFC: $1.040 \cdot 10^{-4}$ ) between $\mathrm{V} 4$ neurons and those V1 subpopulations representing the behaviorally relevant input as opposed to the SFC between the same V4 neurons and the V1 subpopulations representing the irrelevant stimulus. Also across monkeys and recording pairs $(n=22)$, the coherence between V4 spikes and V1 gamma-band LFP was strongly modulated by attention, following the same pattern as the examples and the difference between the attentional conditions as highly significant (Fig. 5C,D; Wilcoxon signed rank test, $p<10^{-4}$ ). The median interareal gamma-band SFC between V4 neurons and its subset of V1 input processing the attended stimulus was more than eight times higher than between V4 neurons and the subset of V1 input processing the nonattended stimulus (Fig. $5 C, D$; attend toward V1 $\mathrm{RF}, \mathrm{SFC}: 4.97 \cdot 10^{-4}$, range $6.80 \cdot 10^{-5}-2.45 \cdot 10^{-3}$; attend out V1 RF, SFC: $5.96 \cdot 10^{-5}$, range $-2.13 \cdot 10^{-4}-5.51 \cdot 10^{-4}$ ). Note that the observed SFC between oscillatory signals does not necessarily imply a flow of information from V4 to V1 but is compatible with information traveling in both directions (Battaglia et al., 2012).

\section{Discussion}

In summary, the results show that the gamma-band PC of V4 neurons with different parts of their V1 input can be strongly modulated in opposite directions by selective attention. It is strong between V4 neurons and the population of their V1 inputs representing the attended stimulus, while simultaneously it is very weak between the V4 neurons and another part of their V1 inputs, representing the behaviorally irrelevant stimulus. Moreover, the results indicate that even individual V4 neurons can switch dynamically between different inputs by modulation of their coupling strength with selected subpopulations of the afferent input. This excludes the possibility that changes in coupling strength between a local V4 population and different parts of its $\mathrm{V} 1$ input reflect changes in the activity distribution over different neurons in the local V4 population, each with a fixed coherence to a set part of the overall V1 input. Similarly, the changes in interareal gamma-band $\mathrm{PC}$ were not just a reflection of gammaband power changes in local V1 populations. Thus, the results show that the effective connectivity of an individual neuron with its synaptic inputs can be modulated by a dynamic mechanism according to the requirements of selective attention-dependent processing.

Our findings provide a mechanistic explanation for how neurons in area V4 can respond selectively to a nonpreferred at- 
tended stimulus, even if another strongly preferred, but unattended stimulus is present within their RFs (Moran and Desimone, 1985; Treue and Maunsell, 1996; Chelazzi et al., 1998; Reynolds et al., 1999). This effect has often been described as "shrinkage of the RF" around the attended stimulus (Reynolds et al., 1999; Womelsdorf et al., 2006), which is exactly what would happen if all but a certain part of the input-providing connections become ineffective. In this case, the response of a neuron would be influenced predominantly by that subset of connections that stays effective. An important advantage of this mechanism is that it renders the partitioning of the input into subsets highly versatile as it is governed by dynamically changeable PC patterns. Such flexibility is of crucial importance, because the composition of afferent synaptic inputs carrying the relevant signals is determined by the position and properties of the stimulus and hence will vary substantially. Organizing selective signal routing based on dedicated neurons (e.g., relay cells) for each of the huge number of possible compositions of relevant synaptic inputs would be much more expensive in terms of the neuronal circuitry required. A further advantage of a mechanism based on the transient gamma-band interactions is the concentration of spikes in time, thereby directly promoting spike timing-dependent plasticity (Markram et al., 1997; Bi and Poo, 1998; Wespatat et al., 2004) and hence associative memory (Lengyel et al., 2005).

Evidence for a dynamic routing mechanism based on gamma-band PC is also delivered by theoretical studies that explain the selective response to the attended stimulus by changes in interareal gamma-band synchronization (Masuda, 2009; Tiesinga and Sejnowski, 2010). In line with the present findings, these models predict gamma-band PC between the input-receiving neurons and the group of input-providing neurons representing the attended stimulus. To reduce the influence of connections from neurons driven by unattended stimuli, they implemented a shift in phase relationships between populations without modifying the strength of the PC. The present findings show instead a major decline in gamma-band PC. However, this also moves afferent spikes out of the phase that allows influencing the postsynaptic neuron most effectively.

Attention-dependent enhancement of gamma-band synchronization has previously been shown within local groups of neurons in visual cortex (Fries et al., 2001; Taylor et al., 2005; for review, see Chalk et
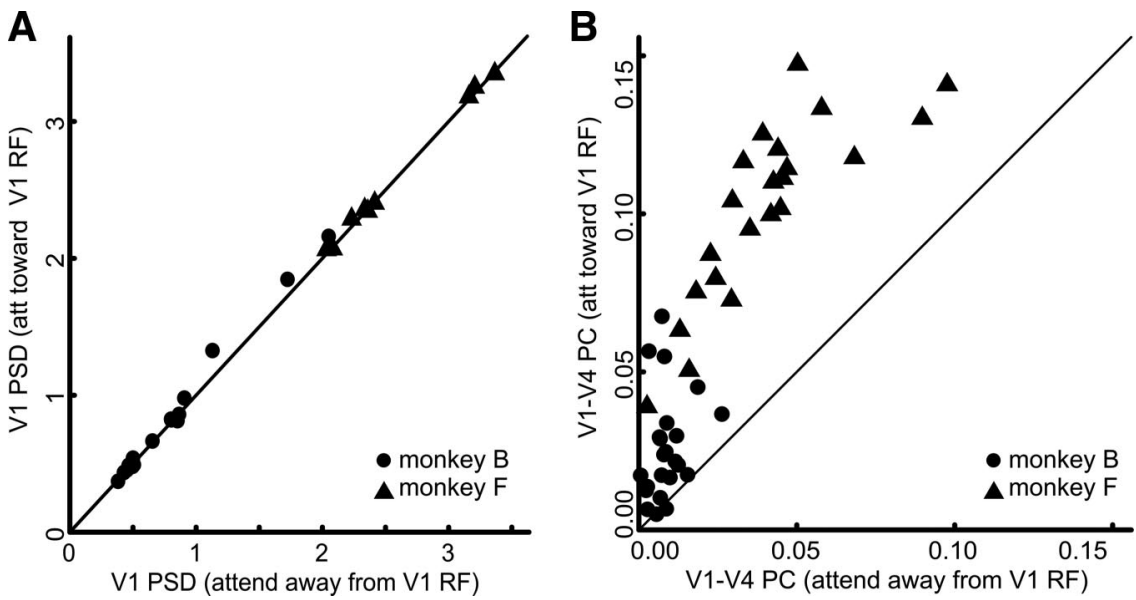

Figure 4. Attentional modulation of gamma-band PC after equalizing V1 PSD between the attentional conditions. $\boldsymbol{A}$, Scatter plot showing the result of the procedure of selecting trials with similar V1 PSD in both attentional conditions. Each dot and triangle represents a single V1 site $(n=24)$. $B$, Scatter plot showing gamma-band PC difference between the attentional conditions. Attentional modulation of V1-V4 gamma-band PC stays similar after equalizing V1 PSD between the attentional conditions. All electrodes that are part of the electrode pairs for the V1-V4 PC coherence were included in the analyses. The TF window over which the PC was calculated was the same as for the original analysis. Each dot and triangle represents a single recording pair $(n=45)$.
A
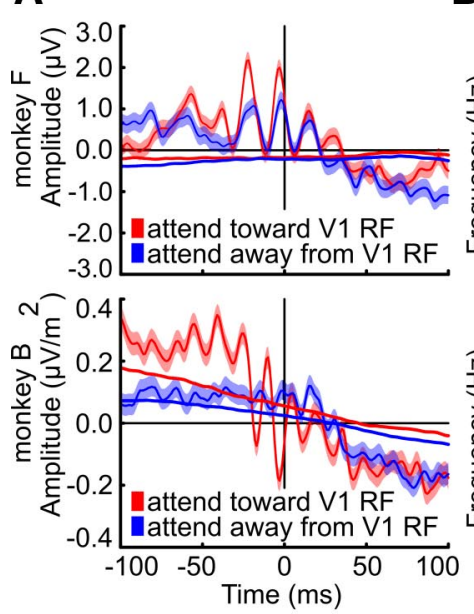

C

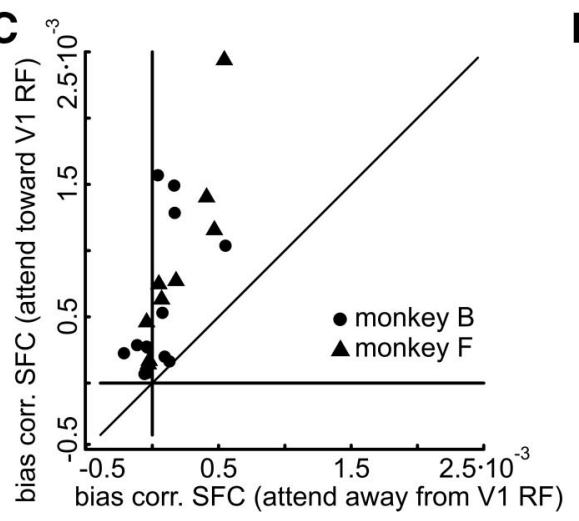

B
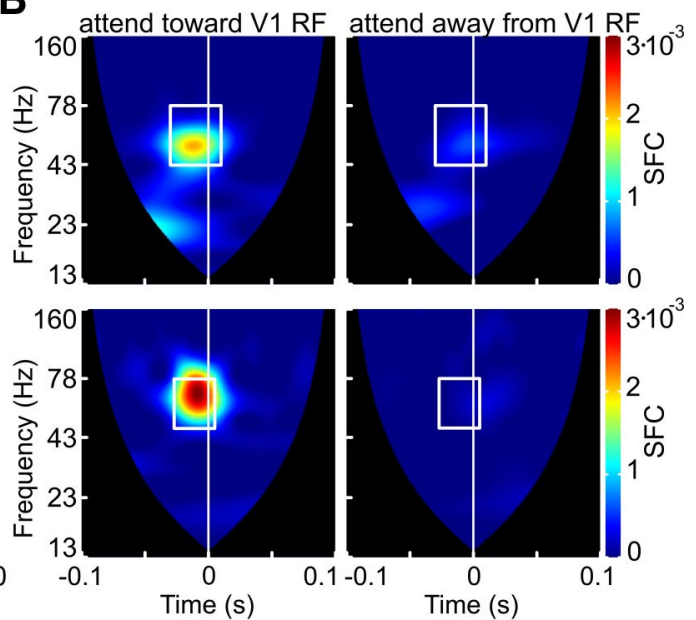

D

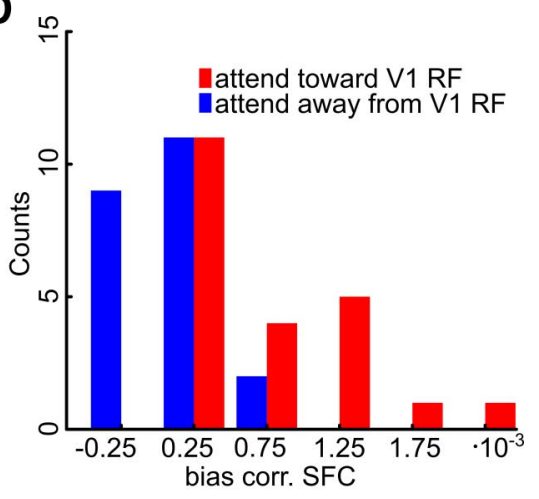

Figure 5. Attention differentially modulates coupling of V4 spikes to gamma-band LFP of multiple V1 subpopulations. $\boldsymbol{A}$, Example STAs of V1 LFPs based on spikes in V4. Upper row monkey F, MUA; lower row monkey B, SUA. Shading represents the standard error, the thin lines represent the STAs after spike jittering. $\boldsymbol{B}$, TF representations of SFC (same pairs as in $\boldsymbol{A}$ ). White boxes delineate the TF window over which the SFC was averaged. Black TF points lie below the cone of influence and cannot be estimated without edge effects. C, Scatter plot showing the V1-V4 gamma-band SFC difference between the attentional conditions. Each dot and triangle represents a single recording pair. $\boldsymbol{D}$, Histograms of the gamma-band SFC (pooled data of both monkeys). 
al., 2010) and was found to have a very close relation to behavior (Taylor et al., 2005). It is therefore thought to be a mechanism that selectively enhances the efficacy of the neuronal signals representing the attended object. An important difference to the routing mechanism described here is that the enhancement of local synchronization is not selective for particular anatomical targets. Any postsynaptic neuron that receives synaptic inputs from neurons in the synchronized group may benefit from spatial summation of the synchronously arriving spikes and hence the enhanced impact of signals representing the attended stimulus. In contrast, the present routing mechanism establishes a signal pathway between a specific sender and a specific receiver population. Only the postsynaptic neurons with gamma-band oscillations that are phase coherent with those of a specific sender population profit from the optimal timing of the afferent synaptic input with respect to the oscillatory modulation of their membrane potential (Lampl and Yarom, 1993; Masuda, 2009; Tiesinga and Sejnowski, 2010).

A potential problem for a routing mechanism based on gamma-band PC was suggested by a recent study showing that the center frequency of gamma-band oscillations in V1 can vary with stimulus contrast (Ray and Maunsell, 2010). It was suggested that this might limit the use of gamma-band oscillations for mechanisms based on PC, since a stimulus could have parts with different contrasts. The resulting different oscillation frequencies could impede synchronization between neurons responding to these different parts. Although in the present study we do not have contrast-variable stimuli, our stimuli are continuously morphing, providing V1 with continuously changing orientations in the RF. This is expected to induce corresponding changes in excitation and inhibition, which has been suggested to be the mechanism underlying the gamma-frequency shift. Nevertheless, we do observe strong attention-dependent V1-V4 PC. In line with this finding, the above-cited study shows that even when two V1 subpopulations of neurons responding to the same object (Gabor grating) engage in gamma-band rhythms with shifted frequencies, there is still significant coherence between them. Furthermore, while the SFC between both V1 populations in the cited study did not show the expected degradation in strength with increasing frequency differences at all, the coherence between the LFPs was found to be somewhat reduced compared with another stimulus condition without frequency shift. The latter may be partly attributed to the fact that the power was also progressively weaker for the shifted case (rendering the phase estimation and hence PC more vulnerable to noise) and partly to the fact that the object versus the ground is less clearly "defined" for the shifted case. Importantly, the observed frequency shifts $(\sim 10 \mathrm{~Hz})$ were limited compared with the bandwidth of the gamma-band oscillations $(\sim 30 \mathrm{~Hz})$, leaving considerable overlap between spectra that allows for synchronization. For "third" neurons, e.g., in V4, any remaining complication is strongly reduced because their average oscillation frequency is likely in the middle of the range spanned by the V1 neurons responding with different frequencies to one object. Hence, the overlap between their spectrum and those of the shifted V1 neurons is much stronger. Thus, the results of the above-cited study are not in conflict with our present findings, which indicate that gamma-band synchronization is particularly suited for routing by phase synchrony.

In the present study we used similar stimuli to create a particularly challenging case for selection. The results show highly selective linking between neurons of areas V1 and V4, whereas the resulting selective response to one of several stimuli inside the RF has been shown for several visual areas (Treue and Maunsell,
1996; Chelazzi et al., 1998; Reynolds et al., 1999). While the known functional anatomy and numerous studies on biased competition leave no doubt that our stimuli placed within the same RF are in a competing constellation and selectively processed in V4 depending on attention, this cannot show up as modulation of firing rate as in classical biased competition paradigms. However, recent measurements using the current task and stimulus constellation showed a good transfer of behaviorally irrelevant brightness modulations of the stimulus to the V4 LFP for the attended but not the nonattended stimulus (D. Rotermund, I. Grothe, S. D. Neitzel, S. Mandon, U. A. Ernst, K. R. Pawelzik, A. K. Kreiter, personal communication). This confirms that competition and attention-dependent selection occurs in our stimulus constellation as predicted by the well established findings described above.

Task-dependent changes of neuronal responses likely to be brought about by differential effective connectivity modulations have been observed for other paradigms (without attention modulations) and other cortical areas (Asaad et al., 2000; Romo et al., 2004; Westendorff et al., 2010). Moreover, interareal synchronization (Engel et al., 1991; Frien et al., 1994) and task-dependent modulation of PC or SFC in the gamma band (Saalmann et al., 2007; Gregoriou et al., 2009) and lower frequency bands (Pesaran et al., 2008) has been observed between different cortical areas previously. Even though none of the studies demonstrated the ability of local populations or even a single neuron to switch between different subsets of its synaptic input, these results suggest that the mechanism described in our study might be a general feature of cortical processing.

In summary, our study provides evidence suggesting that cognitive processes adjust the pattern of effective connectivity by highly selectively modulating gamma-band PC between neurons and different subsets of their input in accordance with quickly changing requirements of cortical information processing (Fries, 2005; Kreiter, 2006). This may be a fundamental mechanism to route signals selectively through the brain and to dynamically configure functional circuits in hard-wired anatomical networks.

\section{References}

Abeles M, Goldstein MH Jr (1977) Multispike train analysis. Proc IEEE 65: 762-773. CrossRef

Aertsen AM, Gerstein GL, Habib MK, Palm G (1989) Dynamics of neuronal firing correlation: modulation of "effective connectivity." J Neurophysiol 61:900-917. Medline

Asaad WF, Rainer G, Miller EK (2000) Task-specific neural activity in the primate prefrontal cortex. J Neurophysiol 84:451-459. Medline

Battaglia D, Witt A, Wolf F, Geisel T (2012) Dynamic effective connectivity of inter-areal brain circuits. PLoS Comput Biol 8:e1002438. CrossRef Medline

Bi GQ, Poo MM (1998) Synaptic modifications in cultured hippocampal neurons: dependence on spike timing, synaptic strength, and postsynaptic cell type. J Neurosci 18:10464-10472. Medline

Chalk M, Herrero JL, Gieselmann MA, Delicato LS, Gotthardt S, Thiele A (2010) Attention reduces stimulus-driven gamma frequency oscillations and spike-field coherence in V1. Neuron 66:114-125. CrossRef Medline

Chelazzi L, Duncan J, Miller EK, Desimone R (1998) Responses of neurons in inferior temporal cortex during memory-guided visual search. J Neurophysiol 80:2918-2940. Medline

Engel AK, Kreiter AK, König P, Singer W (1991) Synchronization of oscillatory neuronal responses between striate and extrastriate visual cortical areas of the cat. Proc Natl Acad Sci U S A 88:6048-6052. CrossRef Medline

Frien A, Eckhorn R, Bauer R, Woelbern T, Kehr H (1994) Stimulus-specific fast oscillations at zero phase between visual areas V1 and V2 of awake monkey. Neuroreport 5:2273-2277. Medline

Fries P (2005) A mechanism for cognitive dynamics: neuronal communica- 
tion through neuronal coherence. Trends Cogn Sci 9:474-480. CrossRef Medline

Fries P, Reynolds JH, Rorie AE, Desimone R (2001) Modulation of oscillatory neuronal synchronization by selective visual attention. Science 291: 1560-1563. CrossRef Medline

Gattass R, Nascimento-Silva S, Soares JG, Lima B, Jansen AK, Diogo AC, Farias MF, Botelho MM, Mariani OS, Azzi J, Fiorani M (2005) Cortical visual areas in monkeys: location, topography, connections, columns, plasticity and cortical dynamics. Philos Trans R Soc Lond B Biol Sci 360: 709-731. CrossRef

Grasse DW, Moxon KA (2010) Correcting the bias of spike-field coherence estimators due to a finite number of spikes. J Neurophysiol 104:548-558. CrossRef Medline

Gregoriou GG, Gotts SJ, Zhou H, Desimone R (2009) High-frequency, long-range coupling between prefrontal and visual cortex during attention. Science 324:1207-1210. CrossRef Medline

Kreiter AK (2006) How do we model attention-dependent signal routing? Neural Netw 19:1443-1444. CrossRef Medline

Lachaux JP, Rodriguez E, Martinerie J, Varela FJ (1999) Measuring phase synchrony in brain signals. Hum Brain Mapp 8:194-208. CrossRef Medline

Lampl I, Yarom Y (1993) Subthreshold oscillations of the membrane potential: a functional synchronizing and timing device. J Neurophysiol 70: 2181-2186. Medline

Lengyel M, Kwag J, Paulsen O, Dayan P (2005) Matching storage and recall: hippocampal spike timing-dependent plasticity and phase response curves. Nat Neurosci 8:1677-1683. CrossRef Medline

Markov NT, Misery P, Falchier A, Lamy C, Vezoli J, Quilodran R, Gariel MA, Giroud P, Ercsey-Ravasz M, Pilaz LJ, Huissoud C, Barone P, Dehay C, Toroczkai Z, Van Essen DC, Kennedy H, Knoblauch K (2011) Weight consistency specifies regularities of macaque cortical networks. Cereb Cortex 21:1254-1272. CrossRef Medline

Markram H, Lübke J, Frotscher M, Sakmann B (1997) Regulation of synaptic efficacy by coincidence of postsynaptic APs and EPSPs. Science 275: 213-215. CrossRef Medline

Masuda N (2009) Selective population rate coding: a possible computational role of gamma oscillations in selective attention. Neural Comput 21:3335-3362. CrossRef Medline

Moran J, Desimone R (1985) Selective attention gates visual processing in the extrastriate cortex. Science 229:782-784. CrossRef Medline

Nunez PL, Srinivasan R, Westdorp AF, Wijesinghe RS, Tucker DM, Silberstein RB, Cadusch PJ (1997) EEG coherency I: statistics, reference electrode, volume conduction, Laplacians, cortical imaging, and interpretation at multiple scales. Electroencephalogr Clin Neurophysiol 103:499-515. CrossRef Medline
Pesaran B, Nelson MJ, Andersen RA (2008) Free choice activates a decision circuit between frontal and parietal cortex. Nature 453:406-409. CrossRef Medline

Ray S, Maunsell JH (2010) Differences in gamma frequencies across visual cortex restrict their possible use in computation. Neuron 67:885-896. CrossRef Medline

Reynolds JH, Chelazzi L, Desimone R (1999) Competitive mechanisms subserve attention in macaque areas V2 and V4. J Neurosci 19: 1736-1753. Medline

Romo R, Hernández A, Zainos A (2004) Neuronal correlates of a perceptual decision in ventral premotor cortex. Neuron 41:165-173. CrossRef Medline

Rubehn B, Bosman C, Oostenveld R, Fries P, Stieglitz T (2009) A MEMSbased flexible multichannel ECoG-electrode array. J Neural Eng 6:036003. CrossRef Medline

Saalmann YB, Pigarev IN, Vidyasagar TR (2007) Neural mechanisms of visual attention: how top-down feedback highlights relevant locations. Science 316:1612-1615. CrossRef Medline

Sun T, Yang ZJ (1992) How far can a random walker go? Phys A Stat Mech Appl 182:599-606. CrossRef

Taylor K, Mandon S, Freiwald WA, Kreiter AK (2005) Coherent oscillatory activity in monkey area $\mathrm{V} 4$ predicts successful allocation of attention. Cereb Cortex 15:1424-1437. Medline

Tiesinga PH, Sejnowski TJ (2010) Mechanisms for phase shifting in cortical networks and their role in communication through coherence. Front Hum Neurosci 4:196. Medline

Treue S, Maunsell JH (1996) Attentional modulation of visual motion processing in cortical areas MT and MST. Nature 382:539-541. CrossRef Medline

Ungerleider LG, Galkin TW, Desimone R, Gattass R (2008) Cortical connections of area V4 in the macaque. Cereb Cortex 18:477-499. Medline

Wespatat V, Tennigkeit F, Singer W (2004) Phase sensitivity of synaptic modifications in oscillating cells of rat visual cortex. J Neurosci 24: 9067-9075. CrossRef Medline

Westendorff S, Klaes C, Gail A (2010) The cortical timeline for deciding on reach motor goals. J Neurosci 30:5426-5436. CrossRef Medline

Womelsdorf T, Anton-Erxleben K, Pieper F, Treue S (2006) Dynamic shifts of visual receptive fields in cortical area MT by spatial attention. Nat Neurosci 9:1156-1160. CrossRef Medline

Womelsdorf T, Schoffelen JM, Oostenveld R, Singer W, Desimone R, Engel AK, Fries P (2007) Modulation of neuronal interactions through neuronal synchronization. Science 316:1609-1612. CrossRef Medline

Zar JH (1999) Biostatistical analysis. Upper Saddle River, NJ: Prentice Hall. 